\title{
Un modèle animal pour le syndrome de Werner
}

Le mécanisme du vieillissement et de la mort prématurée qui sont les principales caractéristiques cliniques du syndrome de Werner suscite toujours un intérêt passionné puisqu'il recèle des éléments essentiels à la connaissance des phénomènes de sénescence. Le gène $W R N$, localisé en $8 \mathrm{p}$ $\left(\mathrm{m} / \mathrm{s}\right.$ 1992, $\left.n^{\circ} 4, p .400\right)$, a été isolé $\left(\mathrm{m} / \mathrm{s}\right.$ 1996, $n^{\circ}$ 6-7, p. 802). Bien que cette maladie récessive soit heureusement peu fréquente, plus d'une dizaine de mutations ont été retrouvées dans diverses populations $(\mathrm{m} / \mathrm{s}$ 1997, $n^{\circ} 3$, p. 411). Les comparaisons avec des gènes analogues retrouvés dans d'autres espèces se sont avérées fructueuses. Ainsi, la forte homologie entre la protéine humaine déduite hWRN et la protéine FFA-1 du xénope qui est, chez cet animal, nécessaire à la formation des foyers de réplication de l'ADN, a prouvé que la fonction hélicase de la protéine WRN était certainement importante dans la réplication de l'ADN (m/s 1998, $n^{\circ} 11$, p. 1267).

Chez la levure, une protéine homologue, Sgs1p, est non seulement nécessaire à la stabilité du génome mais, fait notable, elle interagit avec les topo-isomérases I et II. Or, on connaît le rôle de la topo-isomérase I dans le relâchement de l'ADN et dans l'épissage des ARN prémessagers $\left(m / s\right.$ 1996, $n^{\circ} 8$-9, p. 1029). Grâce aux inhibiteurs spécifiques de ces topo-isomérases: la campothécine pour la I [1] et l'étoposide pour la II [2], il est possible d'étudier leur fonctionnement. Si Sgs1p interagit avec les topo-isomérases, pourquoi ne pas supposer un même mécanisme d'action pour la protéine WRN? Une équipe américaine, qui vient de créer un modèle murin de syndrome de Werner vient d'apporter un élément de réponse [3]. Elle vient de cloner le gène Wrn murin qui se situe, comme on pouvait s'y attendre, dans une région synténique du bras court du chromosome 8 humain. Par recombinaison homologue, des mutations analogues à celles observées chez les malades (c'est-à-dire avec délétions d'exons codant pour les domaines hélicase III et IV) ont été obtenues dans des lignées embryonnaires (ES) et des lignées de fibroblastes embryonnaires (MEF). Après création de souris transgéniques, le nombre de souris $\mathrm{Wrn}^{-/-}$obtenu après croisement est moins élevé que ne le laissait prévoir le calcul théorique, ce qui laisse présumer un désavantage embryonnaire. Ces souris $\mathrm{Wrn}^{-/-}$ne semblent pas présenter d'anomalies au cours de la première année de vie (bien qu'une fibrose myocardique extensive et un lymphome à cellules $\mathrm{T}$ aient été observés, qui sont tout à fait exceptionnels). Il faudra les suivre à plus long terme avant de pouvoir tirer des conclusions.

En ce qui concerne les cultures de cellules in vitro, comme celles des malades atteints de syndrome de Werner, les lignées MEF $\mathrm{Wrn}^{-/-}$ont un potentiel de croissance nettement diminué. Quant aux lignées $\mathrm{Wrn}^{-/-}$, elles ne sont pas plus sensibles, ni aux radiations ionisantes, ni aux UV, ni à la mitomycine. Mais l'élément le plus intéressant de toute cette étude est la nette augmentation de la sensibilité des cellules ES Wrn ${ }^{-/-}$aux inhibiteurs des topo-isomérases: elles sont dix fois plus sensibles à la camptothécine et deux à trois fois plus à l'étoposide que les cellules ES normales $\mathrm{Wrn}^{+/+}$, les hétérozygotes
$\mathrm{Wrn}^{+/-}$ayant une sensibilité intermédiaire.

Enfin, autre constatation en faveur d'une interaction avec les topo-isomérases, en procédant à l'extraction du complexe de réplication multiprotéique de l'ADN, la protéine Wrn normale s'y trouve associée, alors que la protéine mutée se retrouve dans la fraction surnageante parmi les protéines solubles. Une perte de la fonction hélicase empêcherait donc l'interaction avec les topo-isomérases. Bien entendu, étant donné la complexité de la machinerie multiprotéique de la réplication de l'ADN [4], il nous reste encore beaucoup à apprendre sur le rôle du gène $W R N$.

S.G.

1. Morham SG, Kluckman KD, Voulomanos N, Smithies O. Targeted disruption of the mouse topoisomerase I gene by camptothecin selection. Mol Cell Biol 1996; 16 : 6804-9.

2. Wang JC. DNA topoisomerases. Annu Rev Biochem $1985 ; 54: 665-97$.

3. Lebel M, Leder P. A deletion within the murine Werner syndrome helicase induces sensitivity to inhibitors of topoisomerase and loss of cellular proliferative activity. Proc Natl Acad Sci USA 1998 ; 95: 13097-102.

4. Michel N, Baldacci G. Réplication. Med Sci $1998 ; 14: 1422-7$.

Les Universités Pariis 5 et Paris 7 organisent sous la responsabilité de $\mathrm{P}$. Bonfils et $\mathrm{P}$. Tran Ba Huy DIPLOME INTERUNIVERSITARE 1999

PHYSIOPATHOLOGIE EI EXPLORATIONS des FONCTIONS SENSORIELLES ORL

Renseignements et inscriptions : Joëlle Lenoir, service de la scolarité, Faculté Lariboisière-St-Lovis, 10, avenue de Verdun, 75010 Paris et Chantal Barbier, secrétariat ORL, Hôpital Lariboisière, 2, rue Ambroise-Paré, 75010 Paris (Tél. : 01499580 57). 